\title{
Sphenoid Sinusitis Complicated by Pneumococcal Meningitis and an Infectious Aneurysm in the Intracavernous Carotid Artery
}

\author{
Zen Kobayashi ${ }^{1}$, Sakiko Itaya ${ }^{1}$, Yuji Nakamura ${ }^{2}$, Miho Akaza ${ }^{1}$, \\ Hiroyuki Tomimitsu ${ }^{1}$ and Shuzo Shintani ${ }^{1}$
}

\begin{abstract}
A 61-year-old Japanese woman presented with a headache and appetite loss lasting for nine days and was admitted to our hospital, where she was diagnosed with pneumococcal meningitis associated with acute sphenoid sinusitis. While the administration of meropenem and dexamethasone ameliorated the meningitis, right third and sixth nerve palsy suddenly developed 10 days after admission. CT angiography subsequently demonstrated an aneurysm in the cavernous portion of the right internal carotid artery. This is the first reported case of sphenoid sinusitis simultaneously complicated by both pneumococcal meningitis and an infectious aneurysm in the intracavernous carotid artery.
\end{abstract}

Key words: infectious aneurysm, internal carotid artery, pneumococcal meningitis, sphenoid sinusitis, cavernous sinus

(Intern Med 54: 1795-1797, 2015)

(DOI: 10.2169/internalmedicine.54.4462)

\section{Introduction}

The sphenoid sinus is located inferiorly to the pituitary gland, superiorly to the nasopharynx, posteriorly to the ethmoid sinus, anteriorly to the clivus and pons and medially to the cavernous sinus, which contain the internal carotid arteries and third, fourth, fifth and sixth cranial nerves. The sphenoid bone is sometimes thin or absent, and adjacent structures may be separated from the sinus cavity by only a thin mucosal barrier (1).

Sphenoid sinusitis is less common than other sinus infections, with one study reporting sphenoid sinusitis to account for $2.7 \%$ of all sinus infections (1). Major organisms detected in acute cases of sphenoid sinusitis include Staphylococcus aureus and Streptococcus pneumoniae (1). Because the sphenoid sinus is located in close proximity to the meninges and cavernous sinus (1), sphenoid sinusitis sometimes causes meningitis including pneumococcal meningitis (2-4) and cavernous sinus syndrome, in which cranial nerve palsy (5), venous thrombosis (1) and carotid artery aneurysms (6-9) may develop. Sphenoid sinus infection may spread to the meninges or cavernous sinus via direct penetration of the sinus wall or extension along the veins (4). We herein report the first case of sphenoid sinusitis simultaneously complicated by both pneumococcal meningitis and an infectious aneurysm in the intracavernous carotid artery.

\section{Case Report}

A previously healthy 61-year-old Japanese woman presented with a headache and appetite loss. Her past medical history included uterine myoma and osteoporosis, for which she had been taking minodronic acid hydrate and eldecalcitol. She consulted a nearby clinic six days after symptom onset. Upon presentation, she felt pain in the posterior region of the neck and head when she bent her head forward, and her body temperature was $38.0^{\circ} \mathrm{C}$. The administration of loxoprofen and cefditoren pivoxil was started; however, her symptoms remained unchanged, and she therefore consulted our hospital nine days after onset. On a physical examination, her body temperature was $38.6^{\circ} \mathrm{C}$, and slight neck stiff-

${ }^{1}$ Department of Neurology, JA Toride Medical Center, Japan and ${ }^{2}$ Department of Otorhinolaryngology, JA Toride Medical Center, Japan Received for publication November 5, 2014; Accepted for publication November 18, 2014 Correspondence to Dr. Zen Kobayashi, zen@bg7.so-net.ne.jp 

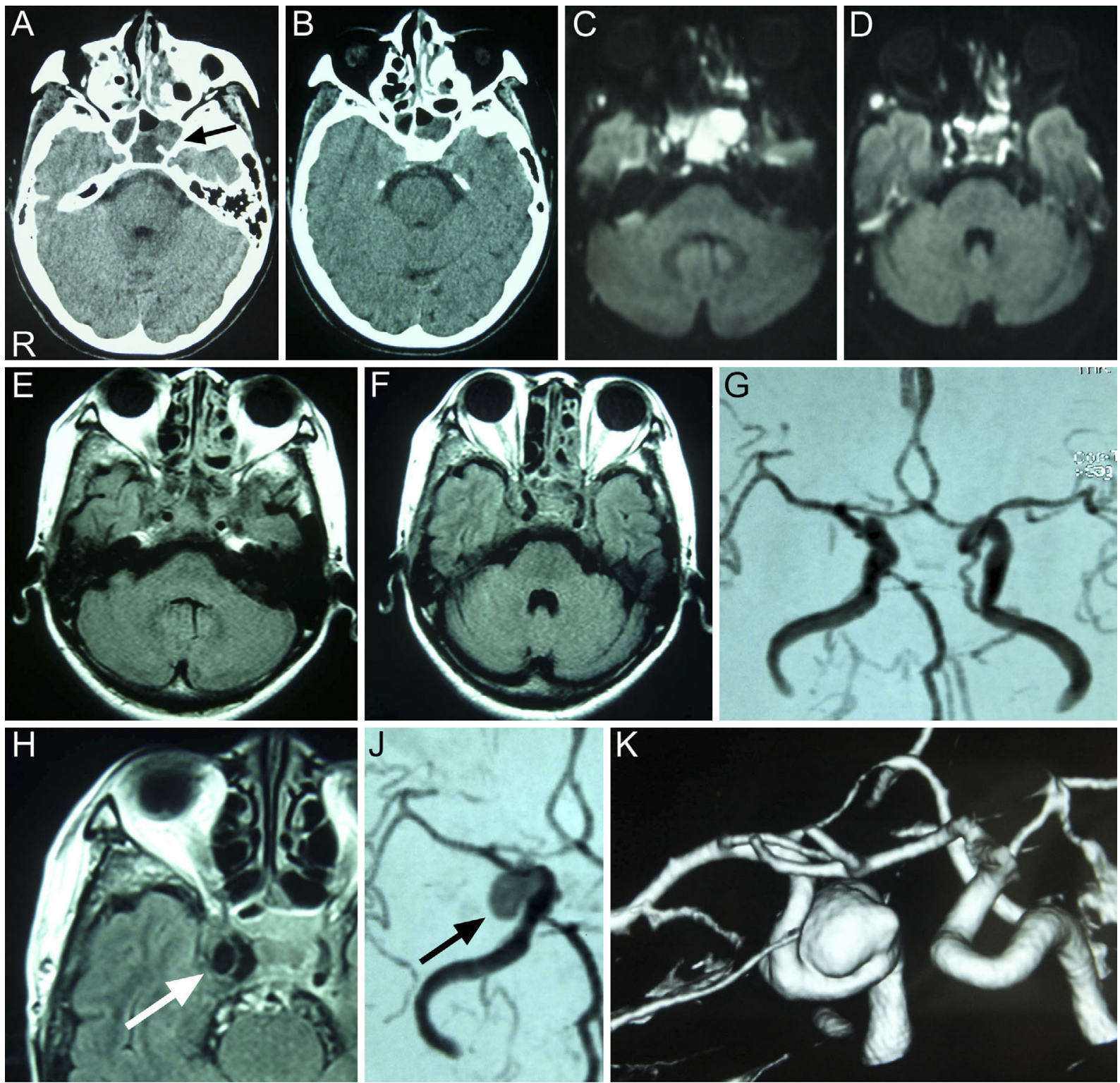

Figure. Brain CT performed on admission demonstrated fluid collection in the bilateral sphenoid sinuses and left ethmoid sinus (A, B). The arrow in (A) indicates the sphenoid sinus. Diffusion-weighted MRI performed one day after admission showed hyperintensity in the bilateral sphenoid sinuses and left ethmoid sinus (C, D). Fluid attenuated inversion recovery (FLAIR) MRI also revealed abnormal intensity within the sphenoid and ethmoid sinuses ( $E, F)$. A normal flow void in the internal carotid arteries was observed $(E, F)$. There were no signs of stenosis or aneurysms in the intracranial arteries on MR angiography (G). FLAIR MRI (H) and MR angiography (J) performed 10 days after admission disclosed an aneurysm in the right internal carotid artery (arrows), and CT angiography ( $K$ ) confirmed the aneurysm in the cavernous portion of the right internal carotid artery measuring approximately $16 \mathrm{~mm}$.

ness was present. There were no signs of a consciousness disturbance or nasal discharge or congestion. Her body weight and height were $48 \mathrm{~kg}$ and $154 \mathrm{~cm}$, respectively. Peripheral blood tests showed a white blood cell count (WBC) of $21,370 / \mu \mathrm{L}$, comprising $2.3 \%$ stab cells, $93.3 \%$ segmented cells, $1.7 \%$ lymphocytes and $2.7 \%$ monocytes, and a Creactive protein (CRP) level of $19.65 \mathrm{mg} / \mathrm{dL}$. Brain CT performed on admission demonstrated fluid collection in the paranasal sinus, including the bilateral sphenoid sinuses (Figure A, B). No bony deficits were observed in the sphenoid bone. Meanwhile, a lumbar puncture carried out on ad- mission revealed an elevated opening pressure $\left(21 \mathrm{~cm} \mathrm{H}_{2} \mathrm{O}\right)$ and yielded watery but slightly yellow fluid exhibiting pleocytosis (cell count: 191/ $\mu \mathrm{L}$, mononuclear cells: $59 / \mu \mathrm{L}$, polynuclear cells: $132 / \mu \mathrm{L}$ ), with a total protein level of 206 $\mathrm{mg} / \mathrm{dL}$ and glucose level of $46 \mathrm{mg} / \mathrm{dL}$ (blood glucose: 143 $\mathrm{mg} / \mathrm{dL}$ ). Gram staining was negative, whereas Streptococcus pneumoniae antigens were positive in both the cerebrospinal fluid (CSF) and urine. The administration of dexamethasone ( $8.25 \mathrm{mg}$, every six hours) and meropenem ( $2 \mathrm{~g}$, every eight hours) was initiated based on a diagnosis of pneumococcal meningitis. On diffusion-weighted MRI performed the next 
day, the fluid within the sphenoid and ethmoid sinuses on CT showed a high intensity (Figure C, D), a finding compatible with the features of the viscous fluid observed in cases of acute sinusitis. Fluid attenuated inversion recovery (FLAIR) MRI also demonstrated abnormal intensity within the sphenoid and ethmoid sinuses (Figure E, F). There was no stenosis or aneurysms in the intracranial arteries on MR angiography (Figure G). Blood and CSF cultures yielded no significant bacteria, possibly due to the prior administration of cefditoren pivoxil. An examination of the nasal cavity using an endoscope performed five days after admission demonstrated purulent discharge from the bilateral sphenoid sinuses, a finding compatible with acute sinusitis, although a culture of the discharge could not be performed. The patient's symptoms gradually resolved with conservative therapy, and a lumbar puncture carried out eight days after admission demonstrated a cell count of $18 / \mu \mathrm{L}$ (mononuclear cells: $2 / \mu \mathrm{L}$, polynuclear cells: $16 / \mu \mathrm{L}$ ), total protein level of $59 \mathrm{mg} / \mathrm{dL}$ and glucose level of $55 \mathrm{mg} / \mathrm{dL}$. However, the patient developed repeated vomiting nine days after admission, followed by right blepharoptosis the next day. A neurological examination performed 10 days after admission showed mydriasis, loss of the light reflex and total ophthalmoplegia in the right eye, indicating right oculomotor and abducens nerve palsy. There were no signs of a sensory disturbance involving the right face. Emergent MRI and MR angiography demonstrated an aneurysm in the right internal carotid artery (Figure H, J), and CT angiography confirmed an aneurysm in the cavernous portion of the right internal carotid artery measuring approximately $16 \mathrm{~mm}$ (Figure K). The patient was subsequently transferred to another hospital, where aneurysm rupture was prevented with coil endovascular embolization.

\section{Discussion}

The occurrence of pneumococcal meningitis (2-4) and intracavernous carotid artery aneurysms (6-9) has been reported separately in patients with sphenoid sinusitis. The latter is thought to result from direct infiltration of the arterial wall by inflammatory cells (8). Based on these reports, we speculate that both the pneumococcal meningitis and intracavernous carotid artery aneurysm detected in our patient were caused by sphenoid sinusitis. The sudden onset of the right third and sixth nerve palsy was thought to be due to rapid enlargement of the intracavernous carotid artery aneurysm (10).

During the course of the disease, our patient did not complain of nasal discharge or congestion suggestive of sinusitis. However, diffusion-weighted MRI clearly demonstrated hyperintensity within the paranasal sinus, reflecting the viscous fluid collection observed in cases of acute sinusitis. Diffusion-weighted MRI may be useful for detecting acute sinusitis in patients with bacterial meningitis. With respect to treatment for sphenoid sinusitis, Lew et al. stated that admission to the hospital with the administration of parenteral antibiotics should be considered in patients complaining of a severe headache (1). The authors also stated that the sphenoid sinus should be drained surgically if the symptoms persist or neurologic signs develop after the initial antibiotic treatment. Nevertheless, in severe cases, such as the present case involving concurrent bacterial meningitis, early drainage of purulent discharge from the sphenoid sinus may be necessary in order to prevent serious complications, including intracavernous carotid artery aneurysms. Regarding the treatment of infectious aneurysms, coil endovascular embolization has been reported to be a useful and minimally invasive approach (9), although the coils may be a potential nidus for persistent infection.

In conclusion, this case is the first reported case of sphenoid sinusitis simultaneously complicated by both pneumococcal meningitis and an infectious aneurysm in the intracavernous carotid artery. Clinicians should be aware of sphenoid sinusitis as a cause of pneumococcal meningitis and intracavernous carotid artery aneurysms.

\section{The authors state that they have no Conflict of Interest (COI).}

\section{Acknowledgement}

The authors are grateful to Dr. Jun-ichi Tsuchiya for evaluating the patient's MRI scans and Drs. Ben Tominaga, Shin Hirota and Mutsumi Fujii for their help with managing the patient.

\section{References}

1. Lew D, Southwick FS, Montgomery WW, Weber AL, Baker AS. Sphenoid sinusitis. A review of 30 cases. N Engl J Med 309: 1149-1154, 1983.

2. Maxwell FC, Couper RT, Morris LL, Goldwater PN, Hansman DJ. Meningitis and sphenoidal sinusitis associated with free gas in the suprasellar cistern. Pediatr Radiol 24: 594-595, 1994.

3. Ohe Y, Maruyama H, Deguchi I, et al. An adult case of pneumocephalus and pneumococcal meningitis associated with the sphenoid sinusitis. Intern Med 51: 1129-1131, 2012.

4. Saitoh A, Beall B, Nizet V. Fulminant bacterial meningitis complicating sphenoid sinusitis. Pediatr Emerg Care 19: 415-417, 2003.

5. Mubaidin AF, Hairi MA. Sixth nerve palsy and sphenoidal sinusitis. Neurosciences (Riyadh) 5: 174-176, 2000.

6. Suzuki N, Suzuki M, Araki S, Sato H. A case of multiple cranial nerve palsy due to sphenoid sinusitis complicated by cerebral aneurysm. Auris Nasus Larynx 32: 415-419, 2005.

7. Nawashiro H, Shimizu A, Shima K, et al. Fatal subarachnoid hemorrhage from an inflammatory cavernous carotid artery aneurysm: failure of conservative treatment after early diagnosis--case report. Neurol Med Chir (Tokyo) 36: 808-811, 1996.

8. Sugie M, Ihihara K, Nakano I, Kawamura M. Infectious aneurysm of the intracavernous carotid artery occurring concomitantly with sphenoid sinusitis; an autopsy case report. J Neurol Sci 278: 115$118,2009$.

9. Hot A, Mazighi M, Lecuit M, et al. Fungal internal carotid artery aneurysms: successful embolization of an Aspergillus-associated case and review. Clin Infect Dis 45: e156-e161, 2007.

10. Linskey ME, Sekhar LN, Hirsch W Jr, Yonas H, Horton JA. Aneurysms of the intracavernous carotid artery: clinical presentation, radiographic features, and pathogenesis. Neurosurgery 26: 71-79, 1990.

(C) 2015 The Japanese Society of Internal Medicine http://www.naika.or.jp/imonline/index.html 